\title{
Ueber Normalen rationaler Raumcurven.
}

\section{(Von Herrn Emil Weyr in Prag.)}

E $_{\mathrm{S} \text { sei }} C_{n}$ eine rationale Raumcurve $n^{\text {ter }}$ Ordnung. Um die durch einen beliebigen Punkt $o$ des Raumes gehenden Normalen der Curve zu erhalten, betrachte man $o$ als Mittelpunkt eines Systems concentrischer Kugeln; es werden dann so viele Normalen der Curve durch den Punkt $o$ gehen, als es Kugeln des Systems giebt, die $C_{n}$ berühren. Und zwar werden die Fusspunkte der Normalen zugleich die Berührungspunkte der tangirenden Kugeln sein.

Das System concentrischer Kugeln ist ein Flächenbüschel zweiten Grades, in welchem die unendlich entfernte Ebene des Raumes doppelt gezählt auch eine Fläche des Büschels darstellt; denn die Flächen haben in der unendlich entfernten Ebene längs des imaginären Kugelkreises einen gemeinschaftlichen Tangentenkegel, dessen Mittelpunkt $o$ ist.

Das System concentrischer Kugeln wird somit auf der rationalen Raumcurve eine Punktinvolution $2 n^{\text {ten }}$ Grades bestimmen*), nämlich jene, welche von den Gruppen der $2 n$ Schnittpunkte der Curve mit den einzelnen Kugeln des Systems gebildet wird. Diese Involution wird somit $2(2 n-1)$ d. i. $4 n-2$ Doppelpunkte besitzen, unter welchen sich jedoch auch die $n$ unendlich entfernten Punkte der Curve $C_{n}$ befinden. Diese unendlich entfernten Punkte sind Doppelpunkte der in Betracht gezogenen Involution, da sie die Schnittpunkte der Curve mit der doppelt gezählten unendlich entfernten Ebene sind und daher jeder von ihnen für zwei einfache Schnittpunkte gilt. Im Endlichen verbleiben somit nur noch $3 n-2$ Doppelpunkte der Involution und jeder von ihnen giebt Veranlassung zu einer berührenden Kugel des concentrischen Systems, und somit zu einer durch $o$ gehenden Normale der Curve $C_{n}$. Wir gelangen daher zu folgendem Ergebniss:

*) Siehe: „Sopra alcune singolarità di second' ordine delle curve gobbe razionali“. Annali di matematica tomo IV. fasc. IV. 
Durch einen beliebigen Punkt des Raumes gehen $3 n-2$ Normalen einer rationalen Raumcurve $n^{\text {ter }}$ Ordnung.

Jede durch $o$ gehende Normale von $C_{n}$ liegt in einer durch $o$ gehenden Normalebene der Curve, und umgekehrt liefert jede durch $o$ gehende Normalebene von $C_{n}$ eine durch denselben Punkt gehende Normale der Curve, so dass wir auch sagen können:

Durch einen beliebigen Punkt des Raumes gehen $3 n-2$ Normalebenen einer rationalen Raumcurve $n^{\text {ter }}$ Ordnung.

Die Normalebenen sind Berührungsebenen der Fläche der Krümmungsaxen und wir haben daher auch den Satz:

Die Fläche der Krümmungsaxen einer rationalen Raumcurve $n^{\text {ter }}$ Ordnung ist von der $3 n-2^{\text {ten }}$ Classe.

Es sei hier bemerkt, dass die Fläche der Krümmungsaxen vom Grade $6(n-1)$ ist.

Dieselben Betrachtungen, welche wir für Raumcurven durchgeführt haben, lassen sich auch für rationale ebene Curven anstellen, und es tritt an die Stelle des concentrischen Kugelsystems ein System concentrischer Kreise.

Prag, im October 1871. 